\title{
Rapid histological changes in endomyocardial biopsy specimens after myocarditis
}

\author{
Anne M Keogh, Margaret E Billingham, John S Schroeder
}

\begin{abstract}
The course and response to treatment in acute lymphocytic myocarditis are conventionally monitored by endomyocardial biopsy performed every 3-12 weeks. A patient with a short history (five days) of acute myopericarditis of unknown aetiology presented in cardiogenic shock with evidence of severe systolic dysfunction on the echocardiogram. The initial biopsy specimen showed histologically unequivocal myocarditis. Repeat endomyocardial biopsy after four days of treatment with steroids and azathioprine showed substantial histological improvement, a reduction in cellular infiltrate and myocardial necrosis, and interstitial fibrosis. Serial biopsies at 2 weeks and then $1,2,4,5,8$, and 14 months after the initial biopsy showed progressive clearing of cellular infiltrate, increasing interstitial fibrosis, and compensatory myocyte hypertrophy by 4 months. At 14 months scattered lymphocytes persisted but myocyte abnormalities had resolved completely. The patient remained symptom free and systolic function was normal during this recovery period.
\end{abstract}

Early endomyocardial biopsy (within one week of diagnosis) may yield useful histological information on the response to treatment in patients with myocarditis. It may not be necessary to wait the customary 3-4 weeks to repeat the biopsy. This case shows the chronology of histological changes and emphasises that a return to normal myocardial function may precede resolution of the histological abnormalities, which may persist in part or may resolve totally after the acute episode.

Division of

Cardiology, Stanford University School of Medicine, Stanford, California, USA

A M Keogh

J S Schroeder

Division of Cardiac

Pathology, Stanford

University School of

Medicine, Stanford,

California, USA

M E Billingham

Correspondence to Dr Anne M Keogh, Cardiac Transplant Unit, St Vincent's Hospital,

Darlinghurst 2010, Sydney, NSW, Australia.
Case report

An 18 year old hispanic girl presented to hospital in October 1986 with a 5 day history of shoulder arthralgia, fevers, and oral ulceration. For 3 days she had experienced vomiting, pleuritic and right upper quadrant pain, and lightheadedness culminating in an episode of presyncope. There were no other associated symptoms. The patient had previously been well and had two uncomplicated pregnancies and deliveries. She used alcohol, nasal cocaine, and marijuana on occasion. On presentation she was pale, unwell, but afebrile with a systolic blood pressure of $60 \mathrm{~mm} \mathrm{Hg}$ and sinus tachycardia (118 beats/min). Jugular venous pressure was elevated to $10 \mathrm{~cm}$. A third heart sound was audible. Lung fields were clear. There was no lymphadenopathy, thyromegaly, or splenomegaly but the liver was palpable $2 \mathrm{~cm}$ below the costal margin. Hypotension failed to improve with a fluid challenge and treatment with dopamine $6 \mu \mathrm{g} / \mathrm{kg} / \mathrm{min}$ was started. Investigations included white cell count $11.2 \quad 10^{9} / 1$ (normal 4-11 $10^{9} / 1$ ) with a normal differential count. The erythrocyte sedimentation rate, platelet count, and serum electrolytes and creatinine were normal. The serum concentration of aspartate transferase was $318 \mathrm{IU} / 1$ $(<35 \mathrm{IU} / 1)$, serum alanine transferase was normal, creatine kinase was $680 \mathrm{IU} / 1$ $(<215 \mathrm{IU} / 1)$ (with the cardiac derived fraction representing $6 \%(0-3 \%))$, and lactate dehydrogenase was $1758 \mathrm{IU} / 1 \quad(<537 \mathrm{IU} / 1)$ with a hepatic/skeletal muscle fraction of $29 \%$ (5-16\%).

The electrocardiogram showed junctional trigeminy interspersed with sinus tachycardia, and left bundle branch block. An electrocardiogram 3 days later showed low voltage sinus tachycardia, poor precordial $\mathbf{R}$ wave development, ST elevation anteriorly, and ST depression laterally. Chest $x$ ray showed blunting of the right costophrenic angle, a normal heart size and contour, and clear lung fields. The left ventricle was diffusely hypo- $\omega$ kinetic on cross sectional echocardiography with an end diastolic diameter of $4.3 \mathrm{~cm}(3 \cdot 7-0$ $5.7 \mathrm{~cm}$ ), end systolic diameter of $3.6 \mathrm{~cm}$ $(<3.2 \mathrm{~cm})$, and evidence of reduced fractional shortening $(16 \%)$ on the $M$ mode echocardiogram. There was a small pericardial effusion. Cardiac valves and wall thickness were normal. A Swan-Ganz catheter was inserted and the right atrial pressure was $\stackrel{D}{\varrho}$ $9 \mathrm{~mm} \mathrm{Hg}$, pulmonary capillary wedge pressure $13 \mathrm{~mm} \mathrm{Hg}$, and cardiac output $3.91 / \mathrm{min}$ on dopamine. 
An endomyocardial biopsy specimen (haematoxylin and eosin stain) showed a dense lymphocytic infiltrate with some plasma cells and eosinophils and myocyte necrosis (fig 1). No viral inclusion bodies, giant cells, granulomas or Aschoff bodies were seen. Specific stains for amyloid protein and iron were negative. The patient was treated with methylprednisolone $500 \mathrm{mg} /$ day followed by a tapered dose of oral prednisone and

Figure 1 Endomyocardial biopsy specimen obtained on day O. (A) The first biopsy showing a diffuse infiltrate (arrows), predominantly of lymphocytes, with myocyte damage $(B)$ (haematoxylin and eosin, original magnification, $\times 80$; inset $\times 320$ ).

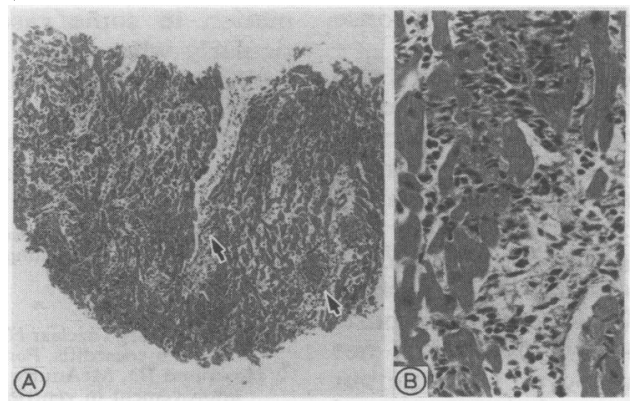

Figure 2 Endomyocardial biopsy specimens obtained on day $4(A)$ and in month 4 (B). The second biopsy (A) showed reduced infiltrate (haematoxylin and eosin; original magnification, $\times 80$ ). (B) $A$ biopsy specimen taken 4 months after the initial biopsy showed interstitial fibrosis $(F)$ with compensatory hypertrophy of the myocytes (haematoxylin and eosin. original magnification, $\times 100)$.

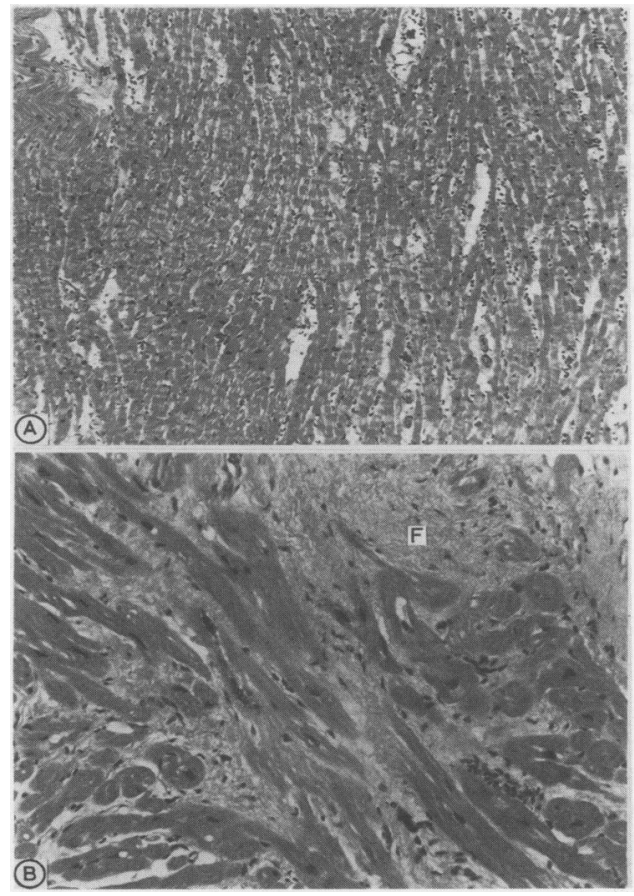

Histological changes with time

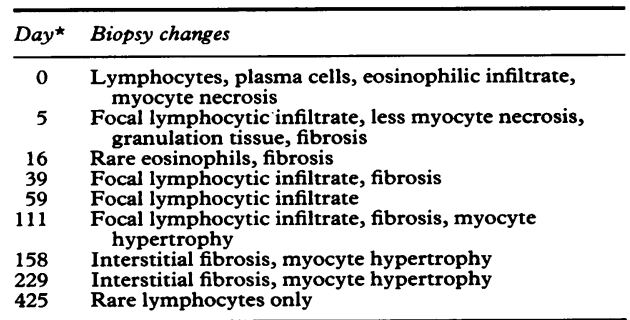

*After diagnosis.

Treatment with methyprednisolone and azathioprine was started 24 hours after the initial cardiac biopsy. azathioprine (75 mg/day). Despite runs of supraventricular tachycardia and second degree Wenckebach phenomenon during the initial 24 hours, the patient was symptom free. Dopamine was stopped by day 5 . A repeat echocardiogram after 48 hours of treatment showed return of systolic function to normal (fractional shortening $29 \%$ ) and pericardial fluid was no longer in evidence. A repeat endomyocardial biopsy after 96 hours of treatment showed greatly reduced cellular infiltrate, myocardial necrosis of a lesser degree, and the new appearance of fibrosis and granulation tissue (fig 2A, table). Serum lactate dehydrogenase and creatine phosphokinase concentrations became normal after 4 days.

The cause of the myocarditis was not ascertained. Viral cultures of blood, sputum, and urine were negative as were serological tests for hepatitis A and B. Epstein-Barr testing was consistent with a past but not recent infection, and streptozyme titre, antinuclear antigen antibody, and rheumatoid factor were all negative. The serum concentration of angiotensin converting enzyme inhibitor was normal. Coxsackie, cytomegalovirus, and echovirus titres were not measured. Culture of a lower lip lesion grew the herpes simplex virus (type 1 ).

At discharge the patient was taking daily doses of prednisone $(0.35 \mathrm{mg} / \mathrm{kg})$ and azathioprine $(1.8 \mathrm{mg} / \mathrm{kg})$ and no specific cardiac medications. Treatment was well tolerated and doses were tapered off to stop at 6 months. She remained symptom free. Pregnancy (not advised) was confirmed 14 months after diagnosis and a normal gestation and delivery ensued.

Six further myocardial biopsies (all without complication) were performed over the ensuing 14 months and showed persistence of nonspecific fibrotic changes and development of myocyte hypertrophy by 4 months (fig $2 B$, table). Echocardiography continued to show a normal chamber size and systolic function. She remained clinically well 3 years after the initial episode.

\section{Discussion}

This patient is of interest in several ways. Firstly, unequivocal biopsy evidence of myocarditis was obtained and immunosuppressive treatment was started within 10 days of the onset of symptoms. Despite considerable myocardial dysfunction at presentation, clinical improvement occurred within 24 hours of the start of treatment. Echocardiographically defined systolic dysfunction reverted to normal by 48 hours, consistent with reduction in myocyte necrosis. Notable points are the rapid development of interstitial fibrosis (within 96 hours) and the early improvement seen in myocardial damage. Despite persistence of a focal lymphocytic infiltrate, fibrosis, and myocyte hypertrophy, systolic function remained normal and the patient remained well.

Acute inflammatory lymphocytic myocarditis can be subacute, recurrent, or chronic. In some patients normal myocardial function is 
restored and others progress to chronic dilated cardiomyopathy. ${ }^{145}$ The role of immunosuppression is unclear-improvement in treated and untreated patients was similar in some studies and in others improvement followed treatment. ${ }^{2356} \mathrm{Up}$ to $40 \%$ of treated patients improve, some returning to normal function, while the remainder die or progress to a chronic dilated cardiomyopathy. ${ }^{3}$ Spontaneous improvement was noted in virtually all series. There is circumstantial evidence in our patient that immunosuppressive treatment may have been beneficial and the response rapid.

Progression of histological changes from those of acute inflammatory lymphocytic myocarditis to interstitial fibrosis, reduction in inflammatory infiltrate, and development of myocyte hypertrophy were reported within 4 weeks of initial diagnosis in treated and untreated patients. ${ }^{1}$ In general, repeat biopsy is advised between 3 weeks and 3 months after the start of treatment. ${ }^{2}$ We know of no reports of biopsy as early as 4 days. We found not only a reduction in lymphocytic infiltrate but also the development of granulation tissue and fibrosis within this short period. This indicates that earlier myocardial biopsy, even within the first week, may show improvement. Myocyte damage was only evident on biopsy specimens taken in the first 14 days. Myocyte hypertrophy was not evident until almost 4 months after the initial biopsy. By 14 months, however, the histological appearance of the specimen had returned to normal and no further biopsies were performed.
The lack of relation between the his- $\frac{T}{D}$ tological severity of myocarditis and clinical outcome has been commented upon before. ${ }^{3}$ In our case too myocardial function was normal despite persisting histological abnormalities. Myocyte hypertrophy, a feature known to be associated with a better prognosis, may have had a favourable influence in this case. Resolution of histological features may lag behind recovery of function.

A repeat biopsy within a week of the start of treatment may give useful histological information in some cases of myocarditis, par- $\overrightarrow{0}$ ticularly where there is clinical improvement and non-invasive evidence of improved car- $\vec{\omega}$ diac function. The histological appearance after myocarditis can return to normal even after relatively long periods of follow up.

AMK was supported by the National Heart Foundation of Australia Overseas Travelling Scholarship.

1 Billingham $\mathrm{M}$, Tazelaar $\mathrm{H}$. The morphological progression f viral myocarditis. Postgrad Med J 1986;62:581-4.

2 Hosenpud JD, McAnulty JH, Niles NR. Lack of objective improvement in ventricular systolic function in patients with myocarditis treated with azathioprine and prednith myocarditis treated with azathiop

3 Dec GW, Palacios IF, Fallon JT, et al. Active myocarditis in the spectrum of acute dilated cardiomyopathies. Clinical features, histologic correlates and clinical outcome. $N$ Engl J Med 1985;312:885-90.

4 Fuster J, Gersh B, Giuliani E, Tajik A, Brandenburg R, Frye $R$. The natural history of idiopathic dilated cardiomyopathy. Am J Cardiol 1981;47:525-31.

5 Mason JW, Billingham ME, Ricci DR. Treatment of acute inflammatory myocarditis assisted by endomyocardial biopsy. Am J Cardiol 1980;45:1037-44.

6 O'Connell JB, Robinson JA, Henkin RE, Gunnar RM. Immunosuppressive therapy in patients with congestive cardiomyopathy and myocardial uptake of gallium-67. Circulation 1981;64:780-6. 